\title{
Clinical and biochemical manifestation of acute viral hepatitis B in Republic of Moldova
}

\author{
Lilia Cojuhari", Victor Pântea', Gheorghe Plăcintă', Valentin Cebotarescu', Olga Chirita', Ana Crudu', Liviu larovoi², \\ Zinaida leseanu', Cristina Marandici ${ }^{1}$ \\ From The 10th Edition of the Scientific Days of the National Institute for Infectious Diseases "Prof Dr Matei Bals" \\ Bucharest, Romania. 15-17 October 2014
}

\section{Background}

Hepatitis B viral represents a pathology with a severe impact on public health. Worldwide, approximately 350 million individuals are chronically infected with hepatitis $B$ virus (HBV). HBV is the leading cause of cirrhosis globally. Once chronic infection is established, approximately $30 \%$ of the patients will develop cirrhosis, and approximately one-quarter of patients with cirrhosis will develop decompensated liver disease within 5 years. Cirrhosis also substantially increases the risk for hepatocellular carcinoma (HCC).

\section{Methods}

In the study were included 73 patients with acute hepatitis $B$ viral with a mean age of $33.20 \pm 1.46$ years that were hospitalized in the Toma Ciorbă Infectious Diseases Clinical Hospital. The analysis parameters: age, sex, onset, clinical manifestation, liver size, disease level, total bilirubin, ALAT, thymol test, prothrombin.

\section{Results}

Acute viral hepatitis B has been observed in the both sexes: women -27 (37\%), men - 46 (63\%). The disease with the acute onset has been manifested in 73 patients $(100 \%)$, more frequently in icteric form in 65 patients (89\%), than in anicteric form in 8 patients (11\%). In $1.4 \%$ of acute HBV patients it occurred in a milder form: in $65.8 \%$ - mild and in $32.8 \%$ - severe form. The preicteric period lasted $7.51 \pm 0.57$ days. Acute B viral hepatitis includes asthenic, dyspeptic and mesenchymal inflammatory syndrome. Biochemical investigations: increased level of bilirubin $184.54 \pm 14.18 \mathrm{mkmol} / \mathrm{L}$, ALT constitutes $11.02 \pm 0.38 \mathrm{mmol} / \mathrm{h} / \mathrm{L}$, thymol test 11.29 $\pm 0.74 \mathrm{U}$ and prothrombin index $-70.99 \pm 1.51 \%$. Hepatomegaly was $3.5 \pm 0.16 \mathrm{~cm}$ in all patients $(100 \%)$, and splenomegaly $-2.0 \pm 0.2 \mathrm{~cm}$ in 39 patients $(53.4 \%)$.

\section{Conclusion}

Acute hepatitis B virus affects both sexes, being more frequent in men, and is manifested through acute onset in the icteric form, the moderate form being characterized clinically by the dyspeptic, asthenic, and biochemical syndrome through the ALT activity increase, bilirubin and thymol test.

\section{Authors' details}

${ }^{1}$ Faculty for Continuing Medical Education, Nicolae Testemițanu State Medical and Pharmacy University, Chişinău, Republic of Moldova. ${ }^{2}$ Department of Infectious Diseases, Parasitology and Tropical Medicine, Nicolae Testemițanu State Medical and Pharmacy University, Chişinău, Republic of Moldova.

Published: 15 October 2014

doi:10.1186/1471-2334-14-S7-P14

Cite this article as: Cojuhari et al: Clinical and biochemical

manifestation of acute viral hepatitis B in Republic of Moldova. BMC Infectious Diseases 2014 14(Suppl 7):P14.

\footnotetext{
* Correspondence: lilia_cojuhari@yahoo.com

${ }^{1}$ Faculty for Continuing Medical Education, Nicolae Testemițanu State

Medical and Pharmacy University, Chişinău, Republic of Moldova

Full list of author information is available at the end of the article
} 